\title{
Assessment of Heart Rate Variability and Heart Rate Turbulence Parameters in the Patients with Arterial Hypertension of the II Degree
}

\author{
Tetyana V. Aleynikova \\ Department of Internal Disease №1 with Course of Endocrinology, Gomel State Medical University, Gomel, 246000, Belarus
}

\begin{abstract}
Background: AH (Arterial hypertension) due to the extremely high incidence is the most important risk factor for development of myocardial infarctions, strokes and lethal outcomes. It is very important to identify a group of patients with increased risk of adverse outcomes to modify treatment and prevention. Significant assistance in resolving this problem is to provide a method of HM (Holter monitoring) which allows estimating the HRV (heart rate variability) and HRT (heart rate turbulence) parameters that are independent risk predictors of sudden cardiac death. The aim of our study was to assess HRV and HRT parameters in patients with AH of the II degree. Materials and methods: We have studied parameters of HRV and HRT in 214 patients with AH of the II-nd degree aged 35 to $70(57.7 \pm 7.6)$ years: 121 women $(56.5 \%)$ and 93 men $(43.5 \%)$. All patients underwent electrocardiography and echocardiography. Statistical analysis of the results has been made using the analytical package Statistics 10.0. Results: The parameters of HRV and HRT in patients with AH of the II degree were studied. Statistically significant correlations of the HRV parameters with the age of patients, average HR, circadian index, and EF (ejection fraction) were revealed. Statistically significant correlations of the HRT parameters with the LVMI (left ventricular mass index), EF, HR average were received. High level of the statistical significance of the interrelation of HRT parameters (TO and TS) has been revealed. Conclusions: The received results can be used during the selection of the group of patients with $\mathrm{AH}$ having high risk for unfavorable outcomes. Taking into account the features of the HRV and turbulence parameters and factors associated with them this will enable the individualization of the assessment of the risk in $\mathrm{AH}$ as much as possible and prescription of adequate treatment for each patient.
\end{abstract}

Key words: AH, HM, HRV, HRT, LVMI, left ventricular ejection fraction.

\section{Introduction}

Publications, devoted to the problem of studying HRV (heart rate variability) in patients with $\mathrm{AH}$ (arterial hypertension) describe some features of the ratio of the tone of sympathetic and parasympathetic parts of the ANS (autonomic nervous system) compared to those in persons with normal BP (blood pressure) $[1,2]$.

Evaluation of HRV changes in patients with $\mathrm{AH}$ allows revealing both increase in sympathetic tone and decrease in parasympathetic tone, which is considered as one of the important mechanisms of formation of

Corresponding author: Tetyana V. Aleynikova, medical doctor, senior lecturer, research fields: cardiology, electrocardiography, Holter monitoring.
AH [3, 4]. Low HRV parameters in hypertensive patients are associated with increased risk of myocardial infarctions, strokes and fatal outcomes. Clinically significant are parameters of time domain analysis of HRV: SDNN (ms) - the standard deviation of NN intervals; SDNN-i (ms) - the mean of the 5-minute standard deviations of $\mathrm{NN}$ intervals calculated over 24 hours; SDANN-i (ms) - the standard deviation of the average NN intervals calculated over short periods, usually 5 minutes; RMSSD (ms) - the square root of the mean of the squares of the successive differences between adjacent NNs.; pNN50 (\%) - the proportion of NN50 divided by total number of NNs [5].

HRT (heart rate turbulence) denotes the baroreflex-mediated short-term oscillation of cardiac 
cycle lengths after spontaneous ventricular premature complexes. Evaluation of HRT after ventricular extrasystoles is currently one of the ways to predict sudden cardiac death in a population or in individuals with cardiovascular disease and allows selecting the high-risk group among patients with myocardial infarction [6, 7]. The most effective is to use HRT in patients with LVEF $>30 \%$. We know two independent parameters of HRT: "onset" (TO) - beginning of turbulence, reflecting the period of tachycardia and "slope" (TS) - slope of turbulence, reflecting the period of bradycardia. Values of TO $<$ $0 \%$ and $\mathrm{TS}>2.5 \mathrm{~ms} / \mathrm{RR}$ are normal, and $\mathrm{TO}>0 \%$ and $\mathrm{TS}<2.5 \mathrm{~ms} / \mathrm{RR}$-abnormal.

The increasing of age is associated with a decrease of HRT. HRT is reduced at high heart rate. As a predictor of risk we can investigate HRT at heart rate $<80 \mathrm{bpm}$. HRT values are usually classified into 3 categories: (1) HRT category 0 means TO and TS are normal; (2) HRT category 1 means 1 of TO or TS is abnormal; and (3) HRT category 2 means both TO and TS are abnormal [8].

During the previous study of HRT in healthy individuals and patients with $\mathrm{AH}$, there was a pronounced difference between the groups in terms of the TO parameter values. The highest mean value was found in healthy individuals and the lowest in patients with $\mathrm{AH}$ of the III degree. Testing of differences between the compared groups using the Mann-Whitney test showed the difference between healthy individuals and patients with $\mathrm{AH}$ of the III degree $(p=0,008890)$ and between patients with $\mathrm{AH}$ of the II degree and AH of the III degree $(p=0$, 037504). The assessment of TS showed more significant differences between healthy individuals and patients with $\mathrm{AH}$ of the III degree ( $p=0,000769)$, between patients with AH II and AH III degree ( $p=0$, 000921) $[9,10]$.

The aim of the present study was to evaluate the parameters of HRV and HRT in patients with hypertension of the II degree. Also the correlations of the parameters of HRV and HRT were analyzed.

\section{Materials and Methods}

The study involved 214 patients with $\mathrm{AH}$ of the II degree. Of these, 121 were women (56.5\%) and 93 men $(43.5 \%)$ aged 35 to $70(57.7 \pm 7.6)$ years. In 173 $(80.8 \%)$, ventricular arrhythmias were recorded according to $\mathrm{HM}$, allowing calculating and estimating the HRT parameters.

The inclusion criteria in the study are AH of the II degree and presence of the sinus rhythm on ECG.

The exclusion criteria from the study are $\mathrm{AH}$ of the I и III degree; symptomatic $\mathrm{AH}$; permanent form of atrial fibrillation; unstable angina at the beginning of the study, stable angina of the III-IV functional class; chronic heart failure III-IV functional class according to the classification of NYHA (New York Heart Association); diabetes mellitus, thyroid gland pathology, pathology of the gastrointestinal tract (chronic ulcer of the stomach and duodenum, ulcerative colitis), organic and functional diseases of the central nervous system; respiratory system diseases (bronchial asthma, chronic obstructive pulmonary disease), acute infectious diseases, exacerbations of chronic diseases.

Instrumental methods of investigation are ECG (electrocardiography), chest X-rays, echocardiography, 24 hours' ambulatory BP monitoring, HM. During the study, Holter was conducted repeatedly. Research was performed without cancellation of antihypertensive therapy received by patients.

We observed patients with $\mathrm{AH}$ of the II degree during the period of $2.6 \pm 1.3$ years and documented the number of myocardial infarctions, strokes, and lethal outcomes occurred during this period. 24 myocardial infarctions, 3 strokes, 4 lethal outcomes have been registered.

Statistical processing of the results was carried out using the software Statistics 10.0. The data are presented as arithmetic mean values and standard deviations $(\mathrm{M} \pm \sigma)$. The significance level of $p<0.05$ 
was considered reliable.

\section{Results}

Patients with $\mathrm{AH}$ of the II degree were divided into four age subgroups (Table 1).

The results of HM in patients with AH of the II degree were evaluated. Diagnostic of autonomic imbalance was carried out by analyzing HRV and HRT parameters. The assessment of time domain HRV parameters in the presented age subgroups was carried out. The assessment was carried out taking into account the normative values of the time domain HRV parameters according to $\mathrm{K}$. Umetani et al. [11] (Table 2).

At the age of 35-39 years, mainly normal and elevated values of HRV parameters were registered. Normal values of pNN50 in the majority of patients indicate a sufficient level of prevalence of the parasympathetic regulation over the sympathetic. Increased SDNN and RMSSD may indicate a compensatory predominance of parasympathetic nervous system tone (in response to increased BP).

At the age of 40-49 years mainly normal values of HRV parameters were recorded. Compared to 35-39-year-old patients the increase of SDNN and
RMSSD was registered in a smaller percentage of cases, which indicates a decrease in the compensatory predominance of the parasympathetic nervous system tone with an increase in the level of BP.

At the 50-59 age normal values of parameters were registered in the majority of patients (SDNN-55.7\%; RMSSD - 46.6\%; pNN50-68.2\%). The increasing of parameters was registered mainly in women, the decreasing - mainly in men.

At the 60-70 age normal values of SDNN were registered in $60 \%$ of cases, RMSSD- $30.5 \%$, pNN50-54.8\%. The registered increase of RMSSD in $61.1 \%$ cases indicates a decrease in the concentration of heart rate due to age-related changes in the reactivity of the ANS.

Assessment of HRT parameters (TO and TS) in four age subgroups was evaluated (Table 3 ).

With age, there is a decrease in the average values of the HRT parameters, especially TO.

The type of the category of HRT parameters estimation in the presented age subgroups was determined (Table 4).

Reduction of the TO parameter was revealed in $15.89 \%$ (34 patients), TS-8.4\% (18 patients), which clarifies the type of category 1 . In $24.29 \%$ category 1

Table 1 Characteristic of the patients with AH of the II degree.

\begin{tabular}{llc}
\hline \multirow{2}{*}{ Age } & \multicolumn{2}{c}{ Distribution of patients with AH by gender and age } \\
\cline { 2 - 3 } & Female & Male \\
\hline $35-39$ & $4(1.9 \%)$ & $3(1.4 \%)$ \\
$40-49$ & $16(7.5 \%)$ & $8(3.8 \%)$ \\
$50-59$ & $54(25.2 \%)$ & $34(15.9 \%)$ \\
$60-70$ & $47(21.9 \%)$ & $48(22.4 \%)$ \\
Totally & $121(56.5 \%)$ & $93(43.5 \%)$ \\
\hline
\end{tabular}

Table 2 Assessment of the time domain HRV parameters.

\begin{tabular}{|c|c|c|c|c|c|c|c|c|c|c|c|c|c|c|c|}
\hline \multirow{4}{*}{ Age } & \multicolumn{15}{|c|}{ Parameters } \\
\hline & \multicolumn{3}{|c|}{ SDNN (ms) } & \multicolumn{3}{|c|}{ SDANNi (ms) } & \multicolumn{3}{|c|}{ SDNNi (ms) } & \multicolumn{3}{|c|}{ RMSSD (ms) } & \multicolumn{3}{|c|}{ pNN50 (\%) } \\
\hline & $\mathrm{N}$ & $\uparrow$ & $\downarrow$ & $\mathrm{N}$ & $\uparrow$ & $\downarrow$ & $\mathrm{N}$ & $\uparrow$ & $\downarrow$ & $\mathrm{N}$ & $\uparrow$ & $\downarrow$ & $\mathrm{N}$ & $\uparrow$ & $\downarrow$ \\
\hline & \multicolumn{15}{|c|}{ Percent (\%) of normal. increased or decreased values of time domain HRV parameters } \\
\hline $35-39$ & 28.6 & 42.8 & 28.6 & 57.1 & 14.3 & 28.6 & 0 & 71.4 & 28.6 & 14.3 & 57.1 & 28.6 & 71.4 & 0 & 28.6 \\
\hline $40-49$ & 70.8 & 12.5 & 16.7 & 91.7 & 0 & 8.3 & 54.2 & 16.7 & 29.1 & 54.2 & 33.3 & 12.5 & 75.0 & 8.3 & 16.7 \\
\hline $50-59$ & 55.7 & 34.1 & 10.2 & 59.1 & 31.8 & 9.1 & 62.5 & 19.3 & 18.2 & 46.6 & 39.8 & 13.6 & 68.2 & 13.6 & 18.2 \\
\hline $60-70$ & 60.0 & 31.6 & 8.4 & 68.4 & 24.2 & 7.4 & 39.5 & 42.1 & 8.4 & 30.5 & 61.1 & 8.4 & 54.8 & 28.4 & 16.8 \\
\hline
\end{tabular}

$\mathrm{N}$-normal value, $\uparrow$-increased, $\downarrow$-decreased. 
Table 3 HRT parameters in patients with AH of the II degree.

\begin{tabular}{llllll}
\hline Age & $35-39(37 \pm 1.6)$ & $40-49(46.5 \pm 3)$ & $50-59(55.2 \pm 2.7)$ & $60-70(64.4 \pm 2.7)$ & $35-70(57.7 \pm 7.6)$ \\
\hline TO $(\mathrm{M} \pm \sigma)$ & $-3.05 \pm 3.42$ & $-1.3 \pm 2.64$ & $-1.29 \pm 3.31$ & $-0.18 \pm 3.65$ & $-0.42 \pm 3.16$ \\
TS $(\mathrm{M} \pm \sigma)$ & $16.06 \pm 13.79$ & $8.13 \pm 4.39$ & $7.89 \pm 6.27$ & $7.18 \pm 7.37$ & $5.62 \pm 4.79$ \\
\hline
\end{tabular}

Table 4 Types of HRT category in patients with AH of the II degree.

\begin{tabular}{lllll}
\hline Age & $35-39$ & $40-49$ & $50-59$ & $60-70$ \\
\hline Category 0 & $6(2.8 \%)$ & $19(8.88 \%)$ & $69(32.24 \%)$ & $60(28.03 \%)$ \\
Category 1 & $1(0.47 \%)$ & $3(1.4 \%)$ & $17(7.94 \%)$ & $31(14.48 \%)$ \\
Category 2 & No & $2(0.94 \%)$ & $2(0.94 \%)$ & $4(1.88 \%)$ \\
\hline
\end{tabular}

Assessment of HRT categories according to A. Bauer et al [7].

was identified. Total reduction of parameters was revealed in $3.74 \%$ of cases ( 8 patients), which clarifies the type of category 2. Pathological values of the parameter TO were identified in $19.63 \%$ of cases (42 patients), TS-12.14\% (26 patients).

The analysis of correlations of the time domain HRV parameters and HRT parameters was evaluated. Correlations with high statistical significance between SDNN and CI $(r=0.531 ; p<0.0001)$; SDNN and TS $(r=0.447 ; p<0.0001)$, SDNN and EF (ejection fraction) $(r=0,53 ; p=0,007)$; RMSSD and TO $(r=$ $0.42 ; p<0.0001)$, RMSSD and TS $(r=0.359 ; p<$ $0.0001)$; pNN50 and TO $(r=0.38 ; p<0.0001)$, pNN50 and TS $(r=0.345 ; p<0.0001)$; pNN50 and $\mathrm{EF}(r=0,41 ; p=0,046)$ were revealed. Statistically significant correlation between SDNN and the age of the patients $(r=-0.2 ; p=0.008)$ was revealed.

A statistically significant correlation between TO and the LVMI $(r=0.22 ; p=0.003)$ was revealed. A significant correlation of TS with the LVMI $(r=-0.21$; $p=0.005$ ) was obtained. Significant correlations of TS with mean HR $(r=-0.28 ; p=0.0002)$, TS and EF $(r=0.2 ; p=0.008)$ were identified.

Association of HRV parameters with the development of lethal outcomes in patients with $\mathrm{AH}$ of the II degree was evaluated: SDNN $(\gamma=-0,7773$; $p=0,00119)$, SDANNi $(\gamma=-0,7026 ; p=0,0034)$, SDNNi $(\gamma=-0,9354 ; p=0,000088), \operatorname{RMSSD}(\gamma=-0,59903 ; p=$ $0,01289)$. Association between TS meanings and lethal outcomes was registrated $(\gamma=-0,90462 ; p=$ 0,000172).
Association of HRT parameters with the development of myocardial infarctions has been identified: TO $(\gamma=0,2768 ; p=0,0144)$, TS $(\gamma=-0,2726$; $p=0,0164$ ).

\section{Discussion}

The majority of patients with $\mathrm{AH}$ of the II degree have normal values of the time domain HRV parameters and HRT parameters. In patients under 40 years there is a predominant increasing of HRV parameters, which may indicate a compensatory predominance of the tone of the parasympathetic nervous system in response to an increasing level of BP. In men over the age of 40 years, the predominance of the sympathetic nervous system tone is more often noted which contributes to a more rapid breakdown of compensatory possibilities and a progressive increasing level of BP.

Pathological changes in HRT are represented mainly by the reduction of the TO parameter, pathological changes in the TS occurred rarer; they are likely to be characteristic of a more pronounced lesion of the autonomous regulation of the heart. It should be noted that the values of the parameters of TO and TS in persons with $\mathrm{AH}$ of the II degree, are within the normative values, with age close to the boundaries of pathological values.

With increasing age of patients, the number of patients with reduction of one parameter or total reduction of turbulence parameters increases.

Statistically significant correlations of HRV 
parameters with patient's age, mean heart rate, circadian index, and EF were revealed. Statistically significant correlations of HRT parameters with the left ventricular myocardial mass index, EF, and mean heart rate were obtained. Significant correlation of TO and TS with the time domain parameters of HRV was identified.

Association between HRV and HRT parameters and the probability of adverse cardiovascular outcomes in patients with $\mathrm{AH}$ of the II degree taken place.

\section{Conclusions}

A comprehensive assessment of HRV and HRT parameters will allow individualizing risk of possible adverse cardiovascular outcomes in $\mathrm{AH}$ and prescribing an adequate treatment.

\section{References}

[1] Grassi, G., Seravalle, G., Quarti-Trevano, F., Dell'Oro, R., Arenare, F., Spaziani, D., et al. 2009. "Sympathetic and Baroreflex Cardiovascular Control in Hypertension-Related Left Ventricular Dysfunction." Hypertension 53: 205-9.

[2] Huikuri, H. V., and Stein, P. K. 2013. "Heart Rate Variability in Risk Stratification of Cardiac Patients." Progress in Cardiovascular Diseases 56 (2): 153-9.

[3] Alieva, A. M., Kopelev, A. M., and Kasatova, T. B. 2004. "Assessment of Heart Rate Variability in Arterial
Hypertension.” Lechebnoe delo 1: 53-9. (in Russian)

[4] Billman, G. E., Huikuri, H. V., Sacha, J., and Trimmel, K. 2015. "An Introduction to Heart Rate Variability: Methodological Considerations and Clinical Applications." Frontiers in Physiology 6 (55): 55.

[5] Huikuri, H. V., and Stein P. K. 2012. "Clinical Application of Heart Rate Variability after Acute Myocardial Infarction.”. Frontiers in Physiology 3:41.

[6] Stein, P. K., and Barzilay, J. I. 2011. "Abnormal Heart Rate Turbulence Predicts Cardiac Mortality in Low; Intermediate and High Risk Older Adults." J. Cardiovasc Electrophysiol 22 (2): 122-7.

[7] Bauer, A., Zürn C. S., and Schmidt, G. 2010. "Heart Rate Turbulence to Guide Treatment for Prevention of Sudden Death.” Journal of Cardiovascular Pharmacology 55 (6): 531-8.

[8] Bauer, A., Malik, M., Schmidt, G., et al. 2008. "Heart Rate Turbulence: Standards of Measurement, Physiological Interpretation, and Clinical Use. International Society for Holter and Noninvasive Electrophysiology Consensus." J. Am. Coll. Cardiology 52 (17): 1353-65.

[9] Dimitriev, D. A., and Tujzarova, I. A. 2010. "Features of Heart Rate Turbulence in Normal and at Arterial Hypertension." Vestnik Chuvashskogo gosudarstvennogo pedagogicheskogo universiteta im 4: 44-9. (in Russian)

[10] Tujzarova, I. A., and Dimitriev, D. 2011. "A Character of Heart Rate Turbulence Depending on the Degree of Essential Hypertension." Vrach-aspirant 47 (4.3): 500-6. (in Russian)

[11] Umetani, K., Singer, D., McCarty, R., et al. 1997. "24 Hour Time Domain Heart Rate Variability and Heart Rate: Relations to Age and Gender over Nine Decades." JACC 31 (3): 593-601. 